\title{
Ocean wave directional spectrum measurement using microwave coherent radar with six antennas
}

\author{
Ze-zong Chen, Lin-gang Fan $^{\mathrm{a})}$, Chen Zhao, and Yan Jin \\ School of Electronic Information, Wuhan University, Wuhan 430079, China \\ a)flg2004@whu.edu.cn
}

\begin{abstract}
An S-band Doppler radar, named MORSE (Microwave Ocean Remote SEnsor), has been developed by Wuhan University to measure ocean wave spectrum. Six narrow beam antennas are equipped to obtain directional wave height spectrum. Each of the antennas has a $3 \mathrm{~dB}$ bandwidth of $30 \mathrm{deg}$, and they are operated in time multiplex mode. The system was applied for a seven-day experiment on the coast of the East China Sea at the end of 2011. Directional wave height spectra are obtained and presented. The time-series of nondirectional wave spectrum and significant wave height fluctuate in the frequency of semidiurnal tide. This signature, consistent with ocean wave properties, suggests that MORSE has been able to measure the directional wave height spectrum.
\end{abstract}

Keywords: ocean wave, wave height spectrum, S-band, radar

Classification: Electron devices, circuits, and systems

\section{References}

[1] M. Hisashi, T. Fukuzo, and S. Toshiko, "Observations of the directional spectrum of ocean waves using a cloverleaf buoy," J. Physic. Oceanography, vol. 5, no. 4, pp. 750-760, 1975.

[2] D. Hauser, K. Kahma, and H. E. Krogstad, Measuring and analysing the directional spectra of ocean waves, Office for Official Publications of the European Communities, Luxembourg, 2005.

[3] W. J. Plant and W. C. Keller, "Evidence of bragg scattering in microwave doppler spectra of sea return," J. Geophys. Res., vol. 95, pp. 16299-16310, 1990.

[4] L. Fan, Z. Chen, and Y. Jin, "Ocean wave measurement by microwave doppler radar," J. Huazhong University of Sci. and Tech. (Natural Science Edition), vol. 40, no. 10 (accepted), 2012.

[5] F. Ding, X. Huang, and B. Wen, "Aliasing radar receiver in FMICW system," IEICE Electron. Express, vol. 7, no. 10, pp. 697-703, 2010.

[6] Z. Chen, C. Zhao, and Y. Jiang, "Wave measurements with multifrequency HF radar in the East China Sea," J. Electromagn. Waves and Appl., vol. 25, pp. 1031-1043, 2011.

[7] E. M. Poulter, M. J. Smith, and J. A. McGregor, "Microwave radar measurements of ocean wave propagation-initial results," Geophys. Res. Lett., vol. 17, pp. 2137-2140, 1990. 
[8] W. J. Plant, W. C. Keller, and K. Hayes, "Normalized radar cross section of the sea for backscatter: 1. mean levels," J. Geophys. Res., vol. 115, C09032, 2010.

[9] M. A. Davidson, T. J. O'Hare, and K. J. George, "Tidal modulation of incident wave heights: fact or fiction?," J. Coastal Res., vol. 2, pp. 151$159,2008$.

\section{Introduction}

Directional wave height spectrum describes the complex signature of windgenerated ocean waves in terms of contributions from waves propagating in different directions with different wavelengths [1]. The importance of directional wave information has long been stressed by the oceanic community. After pioneering contributions to the concept of directional spectrum, many new instruments and analysis techniques have been developed for both in situ observation and for remote sensing. The classical methods such as spatial arrays and pitch-and-roll buoys have been complemented by new technologies such as the displacement and GPS buoys, radars and satellites. However, for decades good measurements of directional wave spectrum were limited almost exclusively to special research campaigns [2].

Microwave coherent radar provides a direct measurement of surface velocity which can be used to remotely measure ocean surface properties. They are originally used to investigate microwave sea scatter, and to deduce modulation transfer functions for marine radar or synthetic aperture radars [3]. Recently, FMCW and pulse Doppler radars were exploited to tentatively calculate ocean wave spectrum from sea surface [4]. However, none of these systems has fully obtained the directional wave height spectrum.

A ground based S-band FMICW radar with six antennas, named MORSE (Microwave Ocean Remote SEnsor), has been developed to measure directional wave spectrum by Wuhan University [4]. The azimuth beam-width of each antenna is 30 degree; hence sea surfaces can be sensed at whole directions. This system was applied for a seven-day experiment at Pingtan which locates at the coast of the East China Sea. In this paper, some preliminary wave results including wave height spectra are extracted from the experimental data sets. In the following, section 2 details the system and the theory; and the results are presented and discussed in section 3 .

\section{System and theory}

MORSE system is operated with the waveform of linear frequency modulation. Range resolving ability is provided by the frequency modulated interrupted continuous wave (FMICW) technique which is able to use the same antenna to translate and receive electromagnetic waves [5]. Range resolution can be readily varied between 5 and $15 \mathrm{~m}$ to suit different ocean status by simply changing the sweep bandwidth [6]. The ocean surfaces up to a maximum distance of $1500 \mathrm{~m}$ can be measured when the transmitted peak power 
is set at $5 \mathrm{~W}$.

Single narrow-beam antenna does not respond to waves arriving from other directions, since when the ocean wave-number forms an angle with the antenna direction which is larger than the directional resolution, the component of the average water particle velocity in the antenna look direction drops to zero [4]. Hence, six narrow-beam antennas (Fig. 1a), each of which covers 30 deg azimuth, are used to measure the full-directional ocean waves which are sum of independent waves with whole directions. The six antennas work in time multiplex mode, and a full six direction scan consumes 18 min (each antenna consumes $3 \mathrm{~min}$ ). With sequentially measurements in six directions, directional wave spectrum can be obtained.

Each antenna divides the sea surface at its azimuth into small patches whose dimensions are smaller than the wavelengths of gravity waves, so the gravity wave orbital motions, which are generally elliptical, can be resolved [3, 4]. In each patch, Doppler velocity is determined by the average movement within the range cell. Provided along the antenna $i$ azimuthal direction there is a gravity wave with angular frequency $\omega$, amplitude $\mathrm{A}(\omega)$ and wave number $K$ (radial and transverse components $k_{r}$ and $k_{\perp}$ ), following Plant et $a l$., in azimuth $\varphi_{i}$ (direction of the antenna $i$ ), the horizontal and vertical components ( $u$ and $w$ ) of radial velocity from a small illuminate footprint at grazing angle $\theta$ are $[4,7]$

$$
\begin{aligned}
& u\left(\varphi_{i}, R, t\right)=U_{0} \cos \left(k_{r} R+k_{\perp} y-\omega t\right)+V_{D}+V_{S} \\
& w\left(\varphi_{i}, R, t\right)=U_{0} \sin \left(k_{r} R+k_{\perp} y-\omega t\right) \times \tanh (K h)\left(1-\frac{V_{D}+V_{S}}{V_{\phi}}\right)
\end{aligned}
$$

Where the orbital speed is

$$
U_{0}=\mathrm{A}(\omega) \omega \operatorname{coth}(K h)
$$

So the radial velocity is given by

$$
V_{r}\left(\varphi_{i}, R, t\right)=u\left(\varphi_{i}, R, t\right) \cos \theta+w\left(\varphi_{i}, R, t\right) \sin \theta
$$

Where $R$ is the distance between radar site and the footprint, $V_{D}$ is the surface current velocity along the visual direction, $V_{S}$ the Bragg scatter velocity, $V_{\phi}$ the long-wave phase velocity, $h$ the water depth, and $y$ the vertical displacement of water particle. Since the grazing angles are typically less than $15 \mathrm{deg}$, the wave height spectral density is $[3,4,7]$

$$
S\left(\varphi_{i}, \omega\right)=\frac{2 V^{2}\left(\varphi_{i}, \omega\right) \tanh (K h)}{g K \cos ^{2} \varphi_{i} \cos ^{2} \theta \cdot \Delta \omega}
$$

Where $V\left(\varphi_{i}, \omega\right)$ is the Fourier transform of $V_{r}\left(\varphi_{i}, R, t\right), g$ is the gravity acceleration and $\Delta \omega$ is the spectral frequency resolution.

The wave height spectrum $S\left(\varphi_{i}, \omega\right)$ collected form one footprint has an 180 deg ambiguity with respect to direction. That means it's not clear that ocean waves in this direction are advancing or receding. To resolve this ambiguity, radial velocities must be collected simultaneously form two closely spaced 
footprints, and then the ambiguity may be resolved by comparing the phase angles of measured radial velocities. Providing the spectra of the velocity series obtained from two neighboring footprints are $V_{1}\left(\varphi_{i}, \omega\right)$ and $V_{2}\left(\varphi_{i}, \omega\right)$ respectively, and $V_{1}\left(\varphi_{i}, \omega\right)$ is the nearer one. The cross spectrum can be calculated as

$$
\psi_{12}\left(\varphi_{i}, \omega\right)=V_{1}\left(\varphi_{i}, \omega\right) \cdot V_{2}^{*}\left(\varphi_{i}, \omega\right)
$$

The ${ }^{*}$ denotes complex conjugate.

The next step is finding the maximum value of $\psi_{12}\left(\varphi_{i}, \omega\right)$, and calculating its phase angle. If the phase angle is positive (means, $0 \sim \pi$ ), the ocean waves are receding, hence $S\left(\varphi_{i}, \omega\right)$ should be changed to $S\left(\varphi_{i}+\pi, \omega\right)$. In the opposite, if the phase angle is negative (means, $-\pi \sim 0$ ), the ocean waves are advancing, and $S\left(\varphi_{i}, \omega\right)$ should not be modified. Note that the footprint spacing must be less than one-half wavelength of the dominant waves.

After a full measurement (with resolving $180 \mathrm{deg}$ ambiguity) over six antennas, directional ocean wave spectrum can be obtained. To increase the azimuth resolution and perfect the directional spectrum, interpolation algorithm in angular domain can be employed. And non-directional spectrum can be obtained from $S\left(\varphi_{i}, \omega\right)$ by

$$
E(\omega)=\sum_{i=1}^{6} S(i, \omega) \cdot \frac{\pi}{6}
$$

Scalar parameters of ocean wave (such as significant wave height and mean wave period) can be computed by the spectral moment analysis [8]. Meanwhile, the dominant wave direction can be obtained according to the spectral energys at six directions.

\section{Results}

\subsection{Directional wave height spectrum}

A seven-day experiment had been done to demonstrate MORSE's feasibility in measuring directional wave height spectrum. This system was deployed at Pingtan on the coast of the East China Sea in the winter of 2011. Halfcircle open sea area is divided into six sectors each of which is illuminated by one antenna. The antenna azimuths are from 100 to $250 \mathrm{deg}$ with stepping interval of 30 deg. Ocean waves over the distances of 200 to $1000 \mathrm{~m}$ away from radar site are measured with the spatial resolution of $5 \mathrm{~m}$.

It takes $3 \mathrm{~min}$ to complete a single direction measurement, and then the radial velocities can be extracted from Doppler spectra. A set of sixdirection velocity series at the range of $700 \mathrm{~m}$ is shown in Fig. 1b, where the mean values are normalized to zero. In each plot Antenna number is marked at the bottom-right. Each of the curves is obtained in $180 \mathrm{~s}$ with interval of $0.5 \mathrm{~s}$. The largest velocities occur at the wave crests, and the propagating nature of ocean wave is apparent in each curve. Since the antenna gives little response to the waves in other directions, the measured velocities fluctuate wildly in dominate-wave direction. During the measurement, the direction of dominant ocean waves are about $150 \mathrm{deg}$, as a result velocity series obtained 


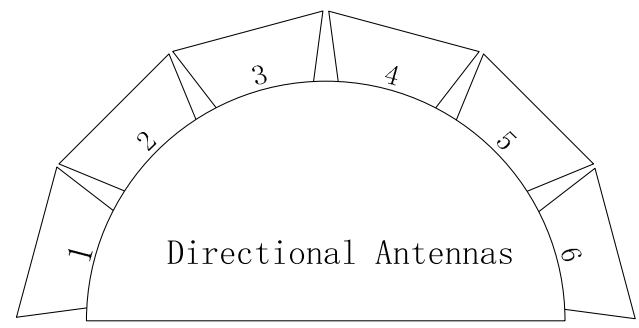

(a)
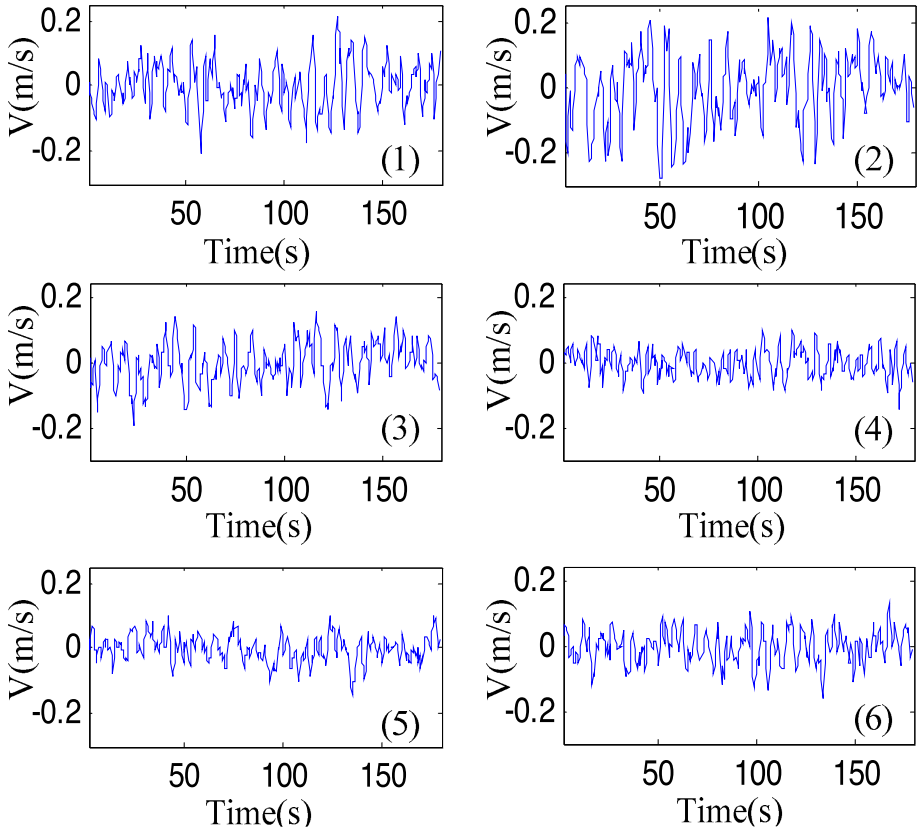

(b)
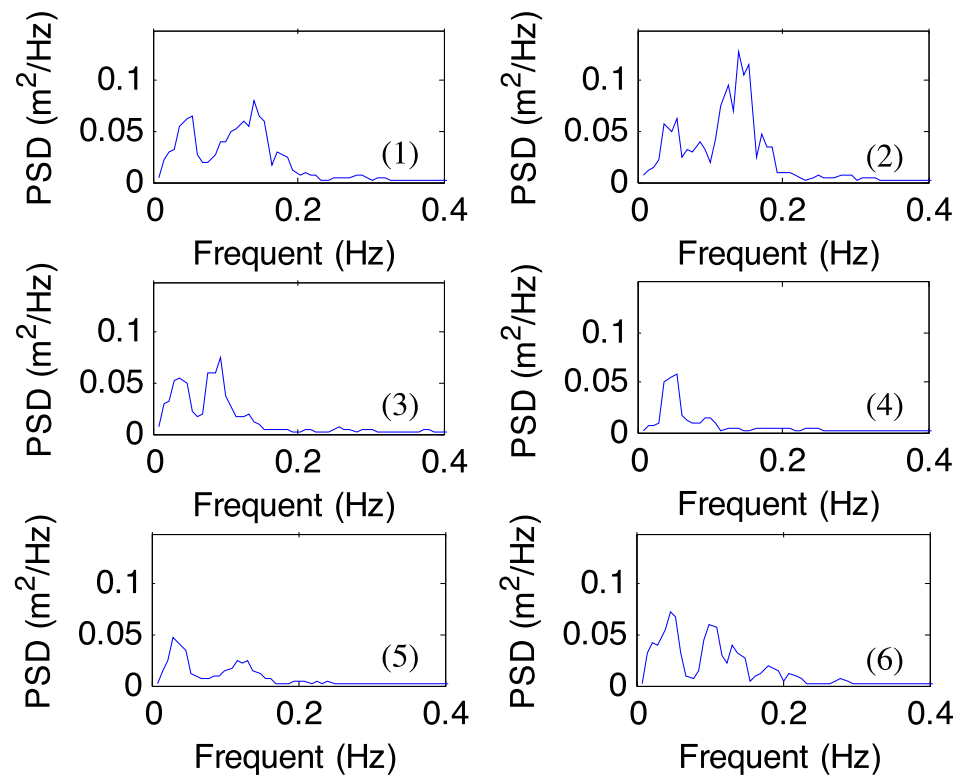

(c)

Fig. 1. Six antennas and their typical results at $700 \mathrm{~m}$. (a) Antenna structure; (b) Radial velocities; (c) Wave height spectrum. Antenna number is marked on each plot. 
by antenna 2 has the largest fluctuation (Fig. 1b(2)). Antennas 4 (Fig. 1b(4)) and 5 (Fig. $1 \mathrm{~b}(5))$, which are almost perpendicular to the dominant wave direction, have velocity series with the smallest variations.

Wave height spectrum can be deduced by spectral analysis of radial velocities according to Eq. (5). The six-direction wave height spectra calculated from the data set in Fig. 1b are shown in Fig. 1c. The spectrum in each direction is obtained from velocity time series of $128 \mathrm{~s}$ and correspondingly the spectral frequency resolution is $0.018 \mathrm{~Hz}$. Similar to the velocity series, the wave height spectrum at dominant wave direction has the largest power spectrum density (Fig. 1c(2)), while the antennas 4 and 5 display lowest density because of their cross-wave directions (Fig. 1c(4), Fig. 1c(5)).

With six direction components, the preliminary directional wave height spectrum can be formed as Fig. 2a. According to Eq. (6) the oceans wave data obtained by antenna $1 \sim 4$ are proved to be from advancing waves, while the other two antennas are corresponding to receding waves. The spectrum components obtained by antenna 5 and 6 should be modified by $180 \mathrm{deg}$ in azimuth, that means their azimuth angles should be changed from 220, 250 deg to 40, $70 \mathrm{deg}$. The directional wave spectrum which has resolved the 180 deg ambiguity is shown in Fig. 2b. To increase the azimuth resolution, cubic spline interpolation is used in angular domian. The interpolated spectrum (Fig. 2c) shows the ocean wave signature more clearly, dominant ocean wave is from around $120 \mathrm{deg}$ and its frequency is about $0.1 \mathrm{~Hz}$.

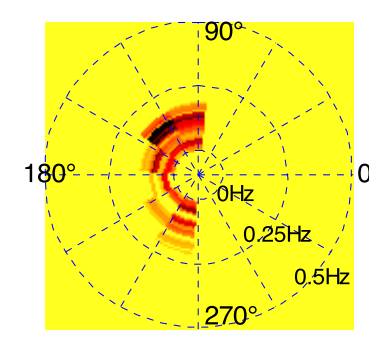

(a)

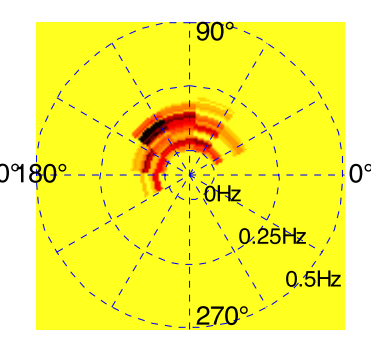

(b)

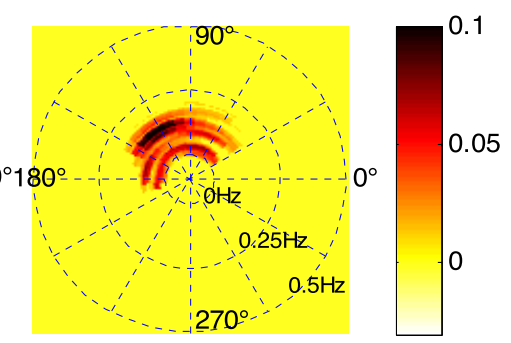

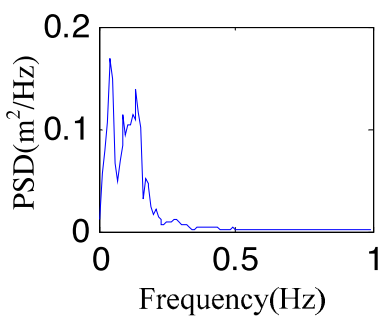

(d) (c)

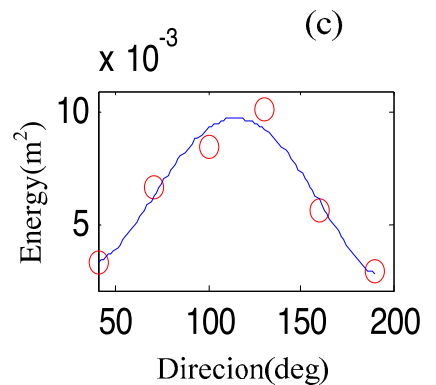

(e)

Fig. 2. The calculated wave spectrum at $700 \mathrm{~m}$. (a) Initial directional spectrum; (b) directional spectrum which has resolved the 180 deg ambiguity; (c) Interpolated directional spectrum; (d) nondirectional spectrum; (e) Six direction spectral energies (circles) and the interpolating result (solid line). 


\subsection{Non-directional wave spectrum and directional distribu- tion}

By Eq. (7) non-directional wave height spectrum can be deduced as Fig. 2d. Most of the spectral energy focuses between 0.04 and $0.15 \mathrm{~Hz}$ which indicates that the dominant wave has an approximate period of $10 \mathrm{~s}$. To estimate the dominant wave direction, polynomial interpolation is employed to the six directional spectral energies. Fig. 2e shows the spectral energies obtained by six antennas (indicated by circles) and the interpolating result (indicated by solid line). The direction degree, corresponding to the maximum energy of the interpolating curve, is 119 deg which is exactly the estimated dominant wave direction.

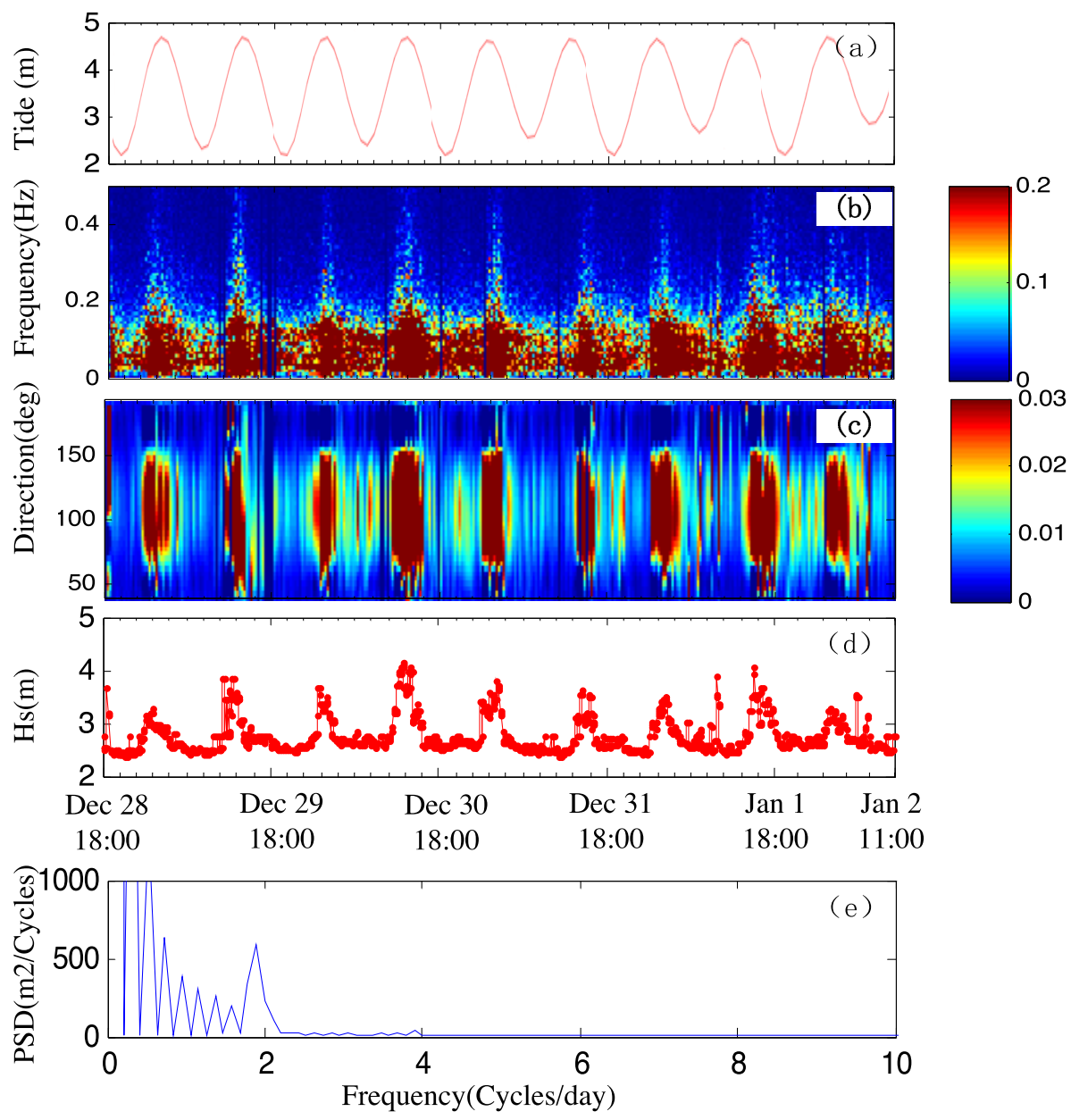

Fig. 3. Tide displacement and measured results at $700 \mathrm{~m}$ for 5 days. (a) Tide displacements at radar site; (b) Non-directional spectrum; (c) Directional distribution of spectral energy; (d) Significant wave height series; (e) Cross-spectrum of wave heights and tide displacements.

The non-directional wave spectra and directional distribution at $700 \mathrm{~m}$ are shown in Fig. 3b and Fig. 3c, with the tidal displacements which are referred to China Marine Environmental Measurement Centre (Fig. 3a). All 
of the non-directional spectra have strong spectral densities between 0 and $0.2 \mathrm{~Hz}$. During high tide, the spectral density grows higher and the spectral frequency band-width grows up to almost to $0.4 \mathrm{~Hz}$. In the plot of directional distribution of spectral energy, ocean wave energy dominantly focuses on about 110 deg which agrees well with the in-situ observation. Furthermore, the strips in Fig3c are in the line of tide displacement. It is clearly seen that the ocean wave spectrum has a synchronous variation with tide.

To investigate the fluctuation more, significant wave height is obtained by the zero order and the first order moment of non-directional spectrum. The results deduced from data in Fig. 3b are shown in Fig. 3d. The variation is clear and significant. A cross-spectral analysis of the significant wave height series and tidal displacement is carried out to study the periodical fluctuation (Fig. 3e). Apart from the intensity near zero frequency which is formed by the mean values, the spectrum has a significant peak at the frequency of 2 cycles/day, which is exactly the principal lunar semi-diurnal frequency. The results show that both the measured wave height spectrum and significant wave height have the fluctuations dominantly caused by semidiurnal tide, which agrees well with the phenomenon that ocean waves are modulated by semidiurnal tide at coastal areas $[4,9]$. We can give the confirmation that the significant wave height has been measured successfully, hence the directional wave height spectrum are measured correctly.

\section{Conclusions}

A microwave Doppler radar with six antennas is developed to measure directional wave height spectrum. Using the measured directional wave height spectra, non-directional wave spectra and direction distributions are calculated. The measured spectra clearly show frequency and direction signatures, which agree well with the visual observation. Both of the deduced wave spectrum and significant wave height fluctuate with semidiurnal tide. The results lead us to make a conclusion that MORSE system with six antennas has the capability of measuring directional wave height spectrum.

\section{Acknowledgments}

This work has been supported by Public Science and Technology Research Funds Projects of Ocean (201205032), 863 High Technology Project of China (2006AA09A303), National Natural Science Foundation of China (40976105) and the Fundamental Research Funds for the Central Universities (20102120201000125, 20082120101000039 and 114003). 